\title{
Modal Dispersion Effects on Coherent Signal Processing Parameters
}

\author{
K. D. FLOWERS
}

\begin{abstract}
Acoustic signals propagated in deep ocean ducts are geometrically (or modally) dispersed. The effects of the dispersion on coherent signal processing is examined in this paper. Using a model, which for long range-deep ocean propagation has been shown to adequately predict dispersion measures, it is determined that the effects of dispersion on the bandwidth of the signal are small. However, in coherently correlating spectrally separated but related signals, the effect on the allowable integration time is significant. Curves relating the allowable integration time as functions of frequency and range rate between the source and receiver are presented.
\end{abstract}

\section{INTRODUCTION}

A COUSTIC signals, when propagated in ducts or refractive media, are geometrically or modally dispersed. For signals propagated in the deep ocean the effects of dispersion are small and for many applications can be ignored. However, if one wishes to coherently process these signals, then dispersion may have a significant effect. This is especially true in coherently processing broadband signal transmissions and signal transmissions received on spatially separated sensors. Assuming that broadband signals can be decomposed into a band of discrete spectral components, the effects of dispersion can be studied by measuring the effects on spectral pairs of components. In this paper we use a standard ocean acoustic propagation model [1] to estimate dispersion induced degradations on coherent processing of spectrally separated signals. This model has previously been used to study the effects of dispersion on $\mathrm{CW}$ signals [2], [3] and has been shown experimentally to adequately predict dispersion in longrange deep-ocean acoustic propagation [4].

\section{Coherent Processing}

To determine the effects of dispersion, we will assume that a distant source transmits two sinusoidal signals that are spectrally separated but have a fixed frequency relationship $\left(f_{2}\right.$ $=q f_{1}, q>1$ ). Since we are only interested in dispersion, the received signals are assumed noise free and of the form

$$
\begin{gathered}
R_{1}(t)=A_{1}(t) \exp \left[i\left(\omega_{1} t+\phi_{1}(t)\right],\right. \text { and } \\
R_{2}(t)=A_{2}(t) \exp \left[i\left(\omega_{2} t+\phi_{2}(t)\right] .\right.
\end{gathered}
$$

We assume that $\omega_{j}=2 \pi(1+\alpha) f_{j}$ for $j=1,2$ where $\alpha$ is the Doppler ratio due to source-receiver motion. Hence $\omega_{2}=$ $q \omega_{1}, q>1$ also holds for the received signals. The quantity of

Manuscript received September 20, 1982; revised April 9, 1985.

The author is with the Acoustics Division, Naval Research Laboratory, Washington, DC 20375 interest is the correlation of these two signals [2]

$$
C(T)=\frac{\left|\int d t R_{1}(t \mid q) R_{2}^{*}(t)\right|}{\left[\int d t\left|R_{1}\right|^{2} \int d t\left|R_{2}\right|^{2}\right]^{1 / 2}}
$$

where $t \mid q$ means that the phase of $R_{1}$ has been multiplied by $q$ and $T$ is the integration interval.

Two parameters of interest when calculating the correlation are the bandwidths of the received signals and the integration time $T$. In the following we will obtain estimates of the effects of dispersion on each parameter and the resulting degradation of the correlation function.

\section{MODEL}

All model calculations used in this paper are fully reported in [2] and [3]. They use the range independent sound speed profile and geometry depicted in Fig. 1. The NRL normal mode model [1] was used where the surface was assumed flat and perfectly reflecting and the bottom was assumed flat and perfectly absorbing. Fig. 2 (Fig. 2 of Ref. 1) is the normal mode evaluation of the acoustic field over the range 1000 $1100 \mathrm{~km}$ for the four frequencies $10,20,40$ and $80 \mathrm{~Hz}$. An estimate of the linear phase variation with range has been removed in order to clearly show the nonlinear phase effects caused by modal dispersion. The model calculations have assumed the source to be at a set of fixed ranges (i.e., no motion). The received signal is then assumed to be equivalent to moving a receiver through the calculated field. Calculations made using modified wave numbers [5] corrected to first order in the ratio of source speed to mode group velocity resulted in negligible changes in the variations of the acoustic field for reasonable source speeds.

\section{ANALYSIS}

The instantaneous frequency of the received signals is of the form

$$
\omega^{\prime}=\omega+\dot{\phi}(t)=\omega+\dot{R} \frac{d \phi}{d R}=\omega\left[1+\frac{\dot{R}}{\omega} \frac{d \phi}{d R}\right]
$$

From Fig. 2 we estimate the maximum of $(1 / \omega) d \phi / d R$ (excluding "instantaneous" jumps of $\pi$ ) to be on the order of 1 $\times 10^{-5} \mathrm{~s} / \mathrm{m}$. Hence, $(\delta f)_{\max } \approx f \dot{R} \times 10^{-5}$ which results in a small dispersion bandwidth for realistic values of $f$ and $\dot{R}$. For example, in long-range deep-ocean propagation, maximum 


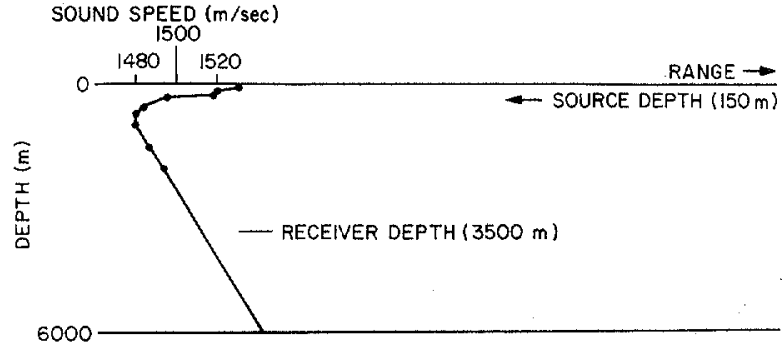

Fig. 1. The geometry and depth dependent sound speed profile used in the acoustic field calculations.
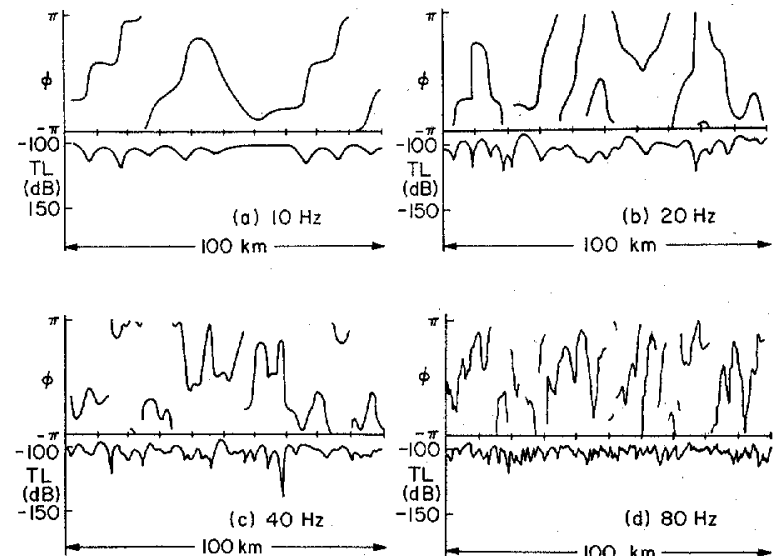

Fig. 2. Calculations of the acoustic field for frequencies of (a) 10 , (b) 20 , (c) 40 , and (d) $80 \mathrm{~Hz}$. The phase of the field with a linear portion removed is illustrated in the top half of each graph. The amplitude of the field (expressed as transmission loss $T L$ in $\mathrm{dB}$ ) is illustrated in the lower half of each graph. The abscissa is range in $\mathrm{km}$ from $1000 \mathrm{~km}$ to $1100 \mathrm{~km}$.

frequencies are on the order of $100 \mathrm{~Hz}$ and expected maximum range rates are of the order of $15 \mathrm{~m} / \mathrm{s}(\approx 30 \mathrm{knots})$, resulting in a maximum bandwidth of $0.03 \mathrm{~Hz}$.

The instantaneous observed frequency ratio $q^{\prime}$ can be written

$$
\begin{aligned}
q^{\prime} & =\frac{\omega_{2}+\dot{\phi}_{2}}{\omega_{1}+\dot{\phi}_{1}}=\frac{\omega_{2}}{\omega_{1}} \frac{\left[1+\left(\dot{R} / \omega_{2}\right) d \phi_{2} / d R\right]}{\left[1+\left(\dot{R} / \omega_{1}\right) d \phi_{1} / d R\right]} \\
& \approx q\left[1+\left(\dot{R} / \omega_{2}\right) d \phi_{2} / d R\right]\left[1-\left(\dot{R} / \omega_{1}\right) d \phi_{1} / d R\right] \\
& \approx q\left[1+\left(\dot{R} / \omega_{2}\right)(d / d R)\left(\phi_{2}-q \phi_{1}\right)\right]
\end{aligned}
$$

where we have used the observed smallness of $(\dot{R} / \omega) d \phi / d R$. Hence

$$
\begin{gathered}
\epsilon=q^{\prime}-q=q\left(\dot{R} / \omega_{2}\right) \frac{d}{d R}\left(\phi_{2}-q \phi_{1}\right), \text { or } \\
\frac{\epsilon}{q \dot{R}}=\frac{1}{\omega_{2}} \frac{d}{d R}\left(\phi_{2}-q \phi_{1}\right) .
\end{gathered}
$$

The latter expression is a measure of the dispersion per unit range rate.

In [2] the distribution of this latter quantity (with $\dot{R}$ in knots) over the range of $50-500 \mathrm{nmi}$ for 15 values of $q(=1.33-8.00)$ was determined. The frequencies used in the calculation were $10,15,20,30,40$, and $80 \mathrm{~Hz}$. From the resulting histograms along with a calculation of the variance of each, it was judged

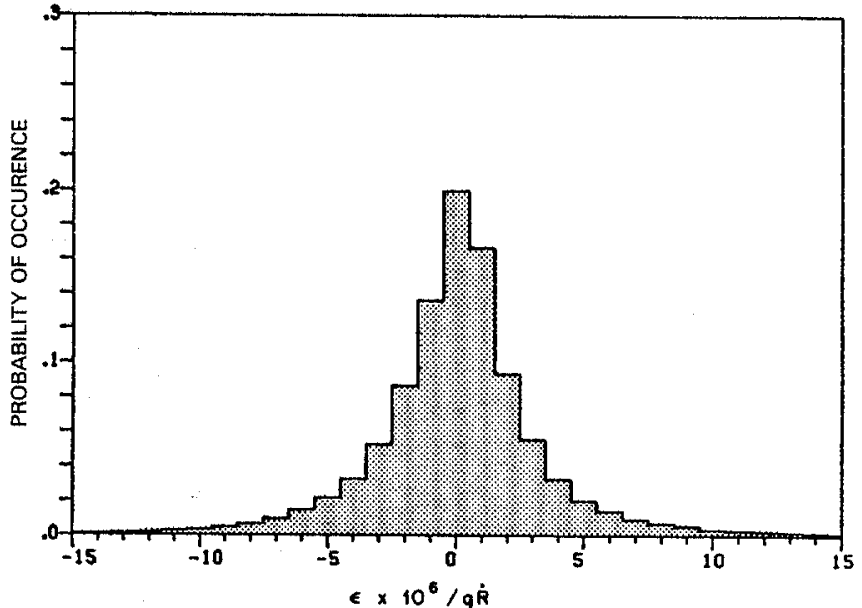

Fig. 3. Histogram depicting the probability density of the spectral dispersion measure $\epsilon$ over the range $50-500 \mathrm{nmi}$. The histogram is an average of 15 histograms developed from the 15 possible ordered frequency pairs selected from $(10,15,20 ; 30,40$, and $80 \mathrm{~Hz})$. The range rate $\dot{R}$ is in knots.

that the difference between them was statistically insignificant. In Fig. 3 (Fig. 42 of Ref. 3 ) is depicted the resulting average histogram of $\epsilon / q \dot{R}$ which is assumed to be independent of $q$ and the signal frequencies used. From (2)

$$
\epsilon / q \dot{R}=\frac{1}{\omega_{2}} \frac{d}{d R}\left(\phi_{2}-q \phi_{1}\right)=\frac{1}{\omega_{2} \dot{R}} \frac{d}{d t}\left(\phi_{2}-q \phi_{1}\right) ;
$$

hence $(d / d t)\left(\phi_{2}-q \phi\right)$ is similarly distributed.

If we assume that over the integration time $T$ the amplitudes of the received signals are constant, then (1) reduces to

$$
C(T)=\frac{1}{T}\left|\int d t \exp \left[i\left(\phi_{2}-q \phi_{1}\right)\right]\right| .
$$

If we further assume that $\phi_{2}-q \phi_{1}=a+b t$ (see discussion below) over the integration time $T$, then

$$
C(T)=\left|\frac{\sin b T / 2}{b T / 2}\right|,
$$

where $b=(d / d t)\left(\phi_{2}-q \phi_{1}\right)$. Thus

$$
\begin{aligned}
E\{C\} & =\sum_{n}\left|\frac{\sin b_{n} T / 2}{b_{n} T / 2}\right| P\left(b=b_{n}\right) \\
& =\sum_{n}\left|\frac{\sin n \gamma}{n \gamma}\right| h(n)
\end{aligned}
$$

where $\gamma=\pi f_{2} \dot{R} T \times 10^{-6}$ and $h(n)$ are the histogram values for $-15 \leq n \leq 15$.

This equation can be numerically solved for the product $f_{2} \dot{R} T$ for specified values of $E\{C\}$. In Fig. 4 we plot $E\{C\}$ versus $f_{2} T(\mathrm{~Hz} \cdot \mathrm{s})$ for several different range rates $\dot{R}$ (knots). From these curves one can determine the integration time $T(\mathrm{~s})$ for an acceptable level of "dispersion degradation" of $E\{C\}$ as a function of the upper frequency $f_{2}$ and range rate $\dot{R}$. In Fig. 5 the integration time $T$ (min) is plotted versus $f_{2} \dot{R}$ $(\mathrm{Hz} \cdot \mathrm{knots})$ for the $E\{C\}=.9, .7$, and .5. From this plot it 


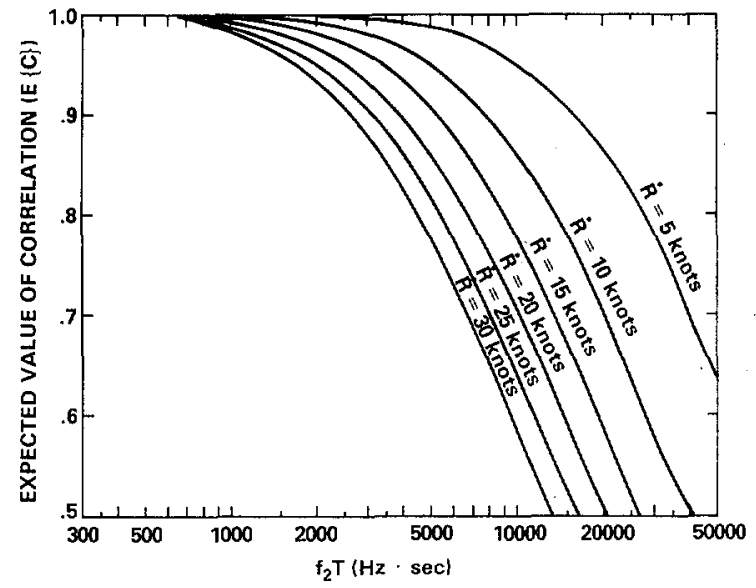

Fig. 4. The expected value of the correlation $(E\{C\})$ is plotted versus $f_{2} T$ with range rate $\dot{R}$ (knots) as a parameter. The frequency $f_{2}$ is the higher of the received pair and $T$ is the integration time in seconds. From this set of curves one can determine the degradation in correlation due to dispersion as a function of the integration time $T$ (assuming $\dot{R}$ and $f_{2}$ known). The general applicability of these curves is limited to $\dot{R} T \leqslant 1 \mathrm{nmi}$ (see discussion in text).

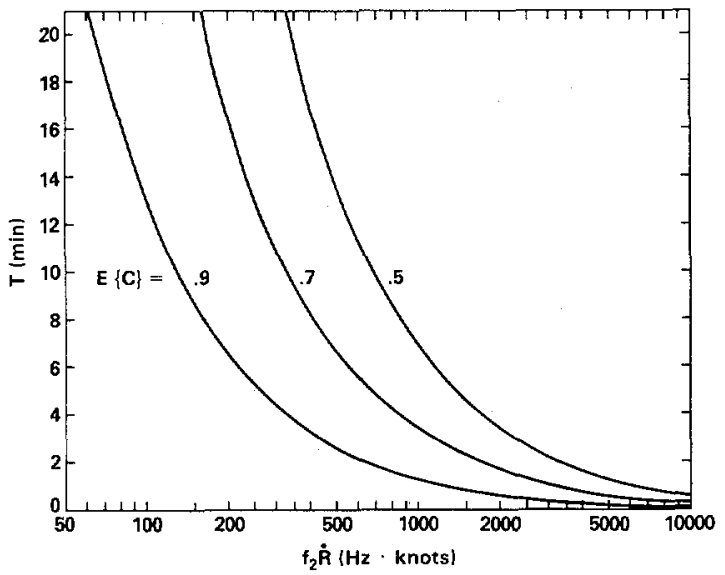

Fig. 5. The integration time $T$ in minutes versus $f_{2} \dot{R}$ for expected values of the correlation $(E\{C\})$. The higher signal frequency $F_{2}$ is in $\mathrm{Hz}$ and the range rate $\dot{R}$ between the source and receiver is in knots. To determine the maximum integration time $T$ for a given situation, calculate the expected range of $f_{2} \dot{R}$ and the amount of degradation, due to dispersion, acceptable in the correlation of the two signals. One then selects the smallest $T$ corresponding to these conditions. The general applicability of these curves is limited to $\dot{R} T \leqslant 1 \mathrm{nmi}$ (see discussion in text).

can be seen that for a given set of parameters $\left(R\right.$ and $\left.f_{2}\right)$ the effects of dispersion on $E\{C\}$ can be quite significant if the integration time $T$ is not chosen properly.

Both Figs. 4 and 5 were developed on the assumption that the phase $\phi_{2}-q \phi_{1}$ was a linear function of time over the integration interval. An examination of plots of $\phi_{2}-q \phi_{1}$ versus range in [3] reveals that, with the exception of isolated points, the linearity assumption is almost always good for range changes of $1 \mathrm{nmi}$ or less. Hence we restrict the applicability of the curves in Figs. 4 and 5 to conditions of $R T$ $\leqslant 1$ nmi.

\section{Summary and Conclusions}

We have evaluated the effects of modal dispersion on acoustic $\mathrm{CW}$ signals propagated in the deep ocean to long ranges. The model used for this evaluation has been experimentally validated. It was found that the effect of dispersion on the bandwidth of the signal was small, but in the coherent processing of two signals, the degradation of the correlation function is highly significant if one uses an improperly long integration interval. This interval can be less than a minute for some signal conditions. The calculations herein considered a source that transmitted two $\mathrm{CW}$ signals with a fixed frequency ratio and reception at a single receiver. However, the effects of dispersion on correlations between signals received on spatially separated receivers (interarray processing) will be similar. These effects will be investigated in future work.

\section{ACKNOWLEDGMENT}

The author expresses his appreciation to Dr. A. A. Gerlach for the many useful comments and suggestions he provided.

\section{REFERENCES}

[1] J. F. Miller and S. N. Wolf, "Modal Acoustic Transmission Loss (MOATL): A Transmission-Loss Computer Program Using a Normal Mode Model of the Acoustic Field in the Ocean," NRL Report 8429; August 1980

[2] K. D. Flowers, "Geometric Dispersion of Acoustic Signals Propagated in a Deep Ocean Channel," Jour. Acoust. Soc. Amer. 73 (3); March 1983.

[3] A. A. Gerlach et al., "Frequency Dispersion of Sound in Undersea Propagation," NRL Report 8600; June 1982.

[4] K. D. Flowers and A. A. Gerlach, "Model Validation of the Geometric Dispersion of Acoustic Signals Propagated in a Deep Ocean Channel," Jour. Acoust. Soc. Amer. 74 (1); July 1983.

[5] A. N. Guthrie et al., "Long-Range Low-Frequenicy CW Propagation in the Deep Ocean: Antigua-Newfoundland," Jour. Acoust. Soc. Amer. 56, 58 (1974).

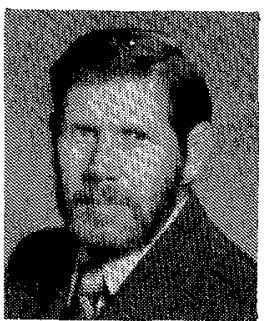

K. D. Flowers received the B.A. degree in mathematics from Southern Illinois University, Carbondale, in 1960 and the M.A. degree in mathematics from Northwestern University, Evanston, IL, in 1964.

Since 1960 he has been a member of the Acoustics Division at the Naval Research Laboratory, Washington, DC. At NRL his work has been concentrated in the field of underwater acoustics and signal processing. He has performed research in the areas of boundary interactions, underwater communications, remote sensing of environmental parameters, coherent, and the dispersive nature of channeled transmissions.

Mr. Flowers is a Fellow of the Acoustical Society of America and a member of Sigma Xi. 